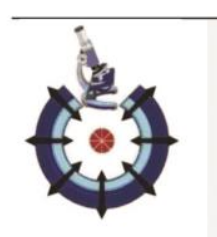

ASRT
Bulletin of Faculty of Science - Zagazig University

https://bfszu.journals.ekb.eg

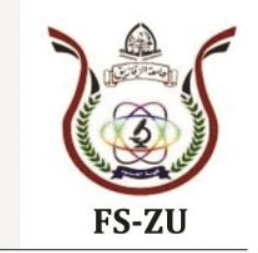

\title{
Studies on the inhibitory activity of some Egyptian natural plant extracts against multidrug resistant bacteria
}

\author{
Mohamed T. Shaaban', Mohamed F. Ghaly², Sara M. Fahmi ${ }^{1 *}$ \\ ${ }^{1}$ Department of Botany and Microbiology, Faculty of Science, Menoufia University, Menoufia, Egypt, \\ ${ }^{2}$ Department of Botany and Microbiology, Faculty of Science, Zagazig University, Zagazig, 44519 Egypt,
}

\begin{tabular}{|c|c|}
\hline ARTICLE HISTORY & Abstract \\
\hline Received: January 2021 & The emergence of types of bacteria resistant to antibiotics is a common and \\
\hline Revised: March 2021 & most dangerous problem in the current era. In this study 50 samples from \\
\hline Accepted: April 2021 & diabetic patients including diabetic foot $(\mathrm{n}=36)$, wounds $(\mathrm{n}=9)$, and \\
\hline KEY WORDS & and MacConkey gar) media. Ten patient's specimens $(20 \%)$ had no \\
\hline Antibacterial activity, & bacterial growth. The sensitivity of 40 bacterial isolates to 7 antibiotics was \\
\hline Natural plant extracts, & performed. A microscopic examination of 40 bacterial isolates by Gram \\
\hline Multidrug resistant bacteria & $\begin{array}{l}\text { stained film was performed. Morphological and biochemical identifications } \\
\text { of selected MDR bacteria were evaluated. The four MDR pathogenic } \\
\text { bacteria are named } S \text {. aureus W, P. aeruginosa DF, K. pneumonia D, and } \\
\text { K. pneumonia BS. Determination of MIC and MBC of the } 3 \text { strongest } \\
\text { antibiotics against } 4 \text { multidrug resistant pathogenic bacteria. Antibacterial } \\
\text { activities of six types of natural plant extracts were studied against tested } \\
\text { pathogenic bacteria. Clove, garlic, and cinnamon boiling water extract } \\
\text { showed inhibitory activity against MDR bacteria. }\end{array}$ \\
\hline
\end{tabular}

*Correspondence: rody.sheref_2013@yahoo.com, 00201021614802.

\section{INTRODUCTION}

The spreading of microorganisms resistant to antimicrobial agents in current years makes it necessary to lock for alternative antimicrobial drugs. Resistant bacteria are known that are not affected by antibacterial drugs as a result of exposure to the antibiotic for a long time due to the changing of bacterial membranes, or due to genetic modifications (Cloete, 2003). It is necessary to isolate, purify, and identify the MDR strains for studying its antibiotic resistance properties and searching for effective antimicrobial therapy. (Harvey and Champe, 2012). Antibiotic is antimicrobial agent has the strength to inhibit or kill the growth of microorganism while doesn't cause harm to the human cell. It is important to examine the susceptibility of the microorganism to the antibiotic to select the most effective one for the treatment. A few methods used for evaluating antibiotics include the filter paper disc (Kirby-Bauer) method (Bauer $\boldsymbol{e t}$ al., 1966), agar and broth dilution method, 
and the dilution method (Owuama, 2015). Minimum inhibitory concentration (MIC) is defined as the lowest concentration of antimicrobial therapy that inhibits microbial growth, as well as Minimum bactericidal concentration (MBC) known as the lowest concentration of antimicrobial therapy required to kill microorganism (Andrews, 2001). Methods that have been used in the determination of MIC and MBC are Macrodilution and microdilution methods (Lambert and Pearson, 2000; Eucast, 2003). Usually, the dilution method (DM) the way for the determination of MIC and MBC (CLSI, 2012).

Medicinal plants that rich in many active compounds like simple phenols, flavonoids, and alkaloids it was found that they have high inhibitory activity against pathogenic microorganisms and can be used as an alternate therapy for microbial infections (Robert et al., 2011; Leon et al., 2001). The existence of the hydroxyl group of phenolic compounds makes these compounds effective as antimicrobial agents; these groups can inhibit the enzyme action of the microorganism. In Egypt, many plants are used today in folk medicine and are sold at herbal vendors and shops (Abdel-Azim et al., 2011). Many medicinal herbs were used by the ancient Egyptians were to treat various diseases. They applied their medicaments in many forms such as powder, pill, and cream form (Bogers et al., 2006). The present study was undertaken to (i) isolate, collect, and identify multidrug resistant bacteria from Egyptian diabetic patients. (ii) Determination of MIC and MBC of the three strongest antibiotics as follows, imipenem, norfloxacin, and nitrofurantoin. (iii) Evaluate the antibacterial activity of some natural medicinal plant extracts.

\section{MATERIALS AND METHODES}

Isolated bacteria were assembled from 50 patients who suffered from diabetes mellitus. From Zagazig University Hospitals.
The samples grew well on Nutrient agar and Blood agar according to (Collee and Marr, 1989), MacConkey agar according to (Koneman et al., 1997).

2.1. Antibiotic susceptibility test: The susceptibility of 40 bacterial isolates to 7 antibiotics was tested by standard disc diffusion technique according to (CLSI 2012). The following antibiotic discs were used: imipenem (IPM 10 $\mu \mathrm{g})$, neomycin $(30 \mu \mathrm{g})$, cefoxitin (30 $\mu \mathrm{g})$ nitrofurantoin $(300 \mu \mathrm{g})$, cefepime (30 $\mu \mathrm{g})$, and amoxicillin/clavulanic acid $(30 \mu \mathrm{g})$ norfloxacin $(10 \mu \mathrm{g})$.

2.2. Microscopic examination of bacterial isolates by (Gram stained film): Preparation of the bacterial smear according to (Collee et al., 1996).

Gram stain by crystal violet solution, gram's iodine solution, decolorizing solution and safranin counterstain according to (Rita et al., 2017). Using oil immersion lens according to (Benson, 1998).

2.3. Identification of bacterial isolates: The biochemical identification of MDR pathogenic bacteria was performed according to (Trujillo and Goodfellow, 2012).

2.4. Determination of the minimum inhibitory concentration (MIC) of the tested antibiotics: MIC of the tested antibiotics was done by preparation of stock solutions, that were prepared from the selected antibiotics (imipenem, norfloxacin, and nitrofurantoin,). According to (Lorian, 2005).

A suitable dilution range was selected before preparing stock solutions. Doubling dilutions were chosen. The concentrations chosen were: 200 $\mathrm{mg} / \mathrm{ml}, \quad 100 \mathrm{mg} \backslash \mathrm{ml}, 50 \mathrm{mg} \backslash \mathrm{ml}, 5$ $\mathrm{mg} \backslash \mathrm{ml}, 500 \mu \mathrm{g} \backslash \mathrm{ml}, 250 \mu \mathrm{g} \backslash \mathrm{ml}, 125$ $\mu \mathrm{g} \backslash \mathrm{ml}, 12.5 \mu \mathrm{g} \backslash \mathrm{ml}, 1.25 \mu \mathrm{g} \backslash \mathrm{ml}, 0.125$ $\mu \mathrm{g} \backslash \mathrm{ml}, 0.0125 \mu \mathrm{g} \backslash \mathrm{ml}$ - Broth cultures were prepared from each of the 4 most 
resistant isolates (isolates number 30, 31, 35, and 40). Tubes containing the broth were then set to $0.5 \mathrm{Mc}$ Farland $[0.05 \mathrm{ml} 1 \%$ barium chloride, and $9.95 \mathrm{ml} 1 \%$ sulfuric acid; this equals a cell density of 1 X $10^{8}$ CFUlml, (Farland, 1987).

Tubes were prepared for each strain of bacteria, each tube consists of one $\mathrm{ml}$ of the antibiotic's concentration just before the required one and one $\mathrm{ml}$ of the diluted broth of the tested isolate, this step is repeated for each strain and each one of the selected antibiotics. Tubes were incubated at $37^{\circ} \mathrm{C}$ for 24 hours. After that, the tubes were noted for turbidity.

2.5. Measurement of the minimum bactericidal concentration (MBC) of the tested antibiotics: The tubes identical to the MIC concentration and the concentrations greater than it in which no turbidity had placed were centrifuged; all organisms were precipitated to the bottom of the tubes. After centrifugation the supernatant consisting of the antibiotic was poured off. Broth without antibiotic was added to the tubes and the organisms taken from the tubes were cultured on nutrient agar and incubated at $37^{\circ} \mathrm{C}$ for 24 hours. The first concentration at which no growth had occurred was MBC (Yamamoto, 2003).

2.6. Preparation of plant extracts: Some natural agents such as clove (Syzygium aromaticum), garlic (Alium sativum), cinnamon (Cinnamomum zylanicum), moringa seeds, moringa green leaves, and moringa dry leaves (Moringa oleifera) extracts were studied for their inhibitory activity against MDR bacteria. The plant samples collected were put to dry in the oven at $45{ }^{\circ} \mathrm{C}$, then pulverized. The powders were kept in closed plastic containers ready for investigation. Seeds and fresh leaves were powdered just before the examination. The dried powdered plant material 20 gm of every plant was extracted with $50 \mathrm{ml}$ of distilled boiled water for 10 minutes and cooled (Izzo et al., 1995). The mixture was boiled in water bath for $15 \mathrm{~min}$ then left at room temperature. All extracts were sterilized by filtration; the filtrate was kept at $4^{\circ} \mathrm{C}$ in the refrigerator till use according to

\section{RESULTS}

Egyptian Pharma Copoeia, (1984).

3.1. Distribution of bacterial isolates among patients: About 50 bacterial isolates were collected from diabetic patients in Zagazig University Hospitals. Firstly, staining reactions and cultural characteristics of the clinical isolates on simple, enriched and selective media were carried out. Fifty samples were analyzed for the presence of pathogenic bacteria on nutrient agar, blood agar and MacConkey agar media. Out of 50 isolates, forty showed bacterial growth, while, ten showed no growth Table (1).

3.2. Antibiotics susceptibility of clinical bacterial isolates: The clinical bacterial isolates were tested for their susceptibility to antibiotic discs using disc diffusion method. The antibiotics, which used in this study, were as following imipenem IPM $(10 \mu \mathrm{g})$, amoxicillin clavulanic acid AMC $(30 \mu \mathrm{g})$, norfloxacin NOR $(10 \mu \mathrm{g})$, cefoxitin FOX $(30 \mu \mathrm{g})$, neomycin $\mathrm{N}$ $(30 \mu \mathrm{g})$, nitrofurantoin $\mathrm{F}(300 \mu \mathrm{g})$ and cefepime FEP $(30 \mu \mathrm{g})$. The isolate No. $30,31,35$, and 40 were resistant to all antibiotic groups (Table 2). The most effective antibiotic against the isolated clinical bacteria was imipenem, followed by norfloxacin and nitrofurantoin, and finally that the more resistant bacterial isolates were detected by cefepime.

3.3. Morphological characteristics and biochemical identification of bacterial isolates: The selected MDR bacteria were identified chemically 
(sugar fermentation, catalase test, coagulase test, hemolysis test, oxidase test, citrate test, $\mathrm{H}_{2} \mathrm{~S}$ tes, $\mathrm{t}$ and in dole test), morphologically and physiologically according to identification protocols as $P$. aeruginosa DF, Klebsiella pneumonia D, Klebsiella pneumonia BS and S. aureus W (Table 3).

3.4. Determination of minimum inhibitory concentration (MIC) and minimum bactericidal concentration (MBC) to three strongest antibiotics for selected bacterial isolates: The most four multidrug resistant bacteria were cultivated with different concentrations of imipenem, norfloxacin, and nitrofurantoin antibiotics in nutrient broth. The results from Table $(\mathbf{4 , 5 )}$ showed the minimum inhibitory concentrations (MIC) of the tested antibiotics and the minimum bactericidal concentrations (MBC).

As shown in Table (4). Imipenem had the highest $\mathrm{MIC}(5 \mathrm{mg} / \mathrm{ml})$ for $P$. aeruginosa followed by MIC (250 $\mu \mathrm{g} / \mathrm{ml}$ ) for $K$. pneumoniae 1 and. Then MIC $(12.5 \mu \mathrm{g} / \mathrm{ml})$ for $K$. pneumoniae 2. Finally, the lowest MIC $(1.25 \mu \mathrm{g} / \mathrm{ml})$ for $S$. aureus. Nitrofurantoin had the highest MIC $(100 \mathrm{mg} / \mathrm{ml})$ for $P$. aeruginosa, $K$. pneumoniae 1 and $S$. aureus Then the lowest MIC $(500 \mu \mathrm{g} / \mathrm{ml})$ for $K$. pneumoniae 2. Norfloxacin had MIC
$(5 \mathrm{mg} / \mathrm{ml})$ for $P$. aeruginosa, $K$. pneumoniae $1, K$. pneumoniae 2 and S. aureus.

The results from Table (5) showed that imipenem had the highest MBC $(100 \mathrm{mg} / \mathrm{ml})$ for $S$. aureus, $K$. pneumoniae 2 and $P$. aeruginosa. Then the lowest MBC $(5 \mathrm{mg} / \mathrm{ml})$ for, $K$. pneumonia 1 . Nitrofurantoin had the highest MBC $(200 \mathrm{mg} / \mathrm{ml})$ for $S$. aureus. Then, lowest MBC $(5 \mathrm{mg} / \mathrm{ml})$ for $K$. pneumoniae 2, and not detected for $K$. pneumoniae 1 and $P$ aeruginosa at concentration $(400 \mathrm{mg} / \mathrm{ml})$. Norfloxacin had the highest MBC $(100 \mathrm{mg} / \mathrm{ml})$ for $S$. aureus. Then the lowest MBC $(50 \mathrm{mg} / \mathrm{ml})$ for $K$. pneumoniae 2 , and not detected for $K$. pneumoniae 1 and $P$. aeruginosa at the concentration $(400 \mathrm{mg} / \mathrm{ml})$.

3.5. Antibacterial activities of some plants boiling water extract on MDR bacteria: The inhibitory effect of plant extracts against MDR bacteria is given in (Table 6). Cinnamon boiling water extract showed the higher inhibitory against all tested bacteria IZDs reached (8-13) $\mathrm{mm}$ followed by clove and garlic, while the inhibitory activity of moringa dry leaves, moringa seeds and moringa green leaves extracts were had no effect against the tested pathogenic bacteria. 
Table (1): The Distribution of most clinical bacterial isolates among studied group of patients.

\begin{tabular}{|c|c|c|c|c|c|}
\hline $\begin{array}{c}\text { No of } \\
\text { patients }\end{array}$ & Age & Gender & $\begin{array}{c}\text { Source of } \\
\text { sample }\end{array}$ & $\begin{array}{c}\text { Fasting } \\
\text { Glucose level }\end{array}$ & Medication \\
\hline 1 & 42 & Female & Diabetic foot & 200 & Insulin \\
\hline 2 & 54 & Male & Diabetic foot & 180 & Insulin \\
\hline 3 & 73 & Male & Diabetic foot & 314 & Tablet \\
\hline 4 & 57 & Female & Bed sores & 250 & Insulin \\
\hline 5 & 50 & Female & Diabetic foot & 150 & Insulin \\
\hline 6 & 67 & Female & Diabetic foot & 230 & Insulin \\
\hline 7 & 80 & Female & Wound & 250 & Insulin \\
\hline 8 & 60 & Female & Diabetic foot & 270 & Insulin \\
\hline 9 & 48 & Female & Wound & 200 & Insulin \\
\hline 10 & 55 & Male & Wound & 185 & Insulin \\
\hline 11 & 63 & Female & Diabetic foot & 300 & Insulin \\
\hline 12 & 60 & Female & Diabetic foot & 210 & Insulin \\
\hline 13 & 53 & Female & Diabetic foot & 130 & Tablet \\
\hline 14 & 46 & Male & Diabetic foot & 170 & Tablet \\
\hline 15 & 68 & Male & Diabetic foot & 170 & Insulin \\
\hline 16 & 60 & Male & Diabetic foot & 500 & Insulin \\
\hline 17 & 61 & Male & Wound & 223 & Insulin \\
\hline 18 & 71 & Male & Bed sores & 300 & Insulin \\
\hline 19 & 67 & Male & Diabetic foot & 230 & Insulin \\
\hline 20 & 60 & Female & Wound & 200 & Insulin \\
\hline 21 & 65 & Female & Diabetic foot & 180 & Insulin \\
\hline 22 & 58 & Female & Diabetic foot & 274 & Insulin \\
\hline 23 & 52 & Male & Diabetic foot & 217 & Insulin \\
\hline 24 & 81 & Male & Diabetic foot & 416 & Insulin \\
\hline 25 & 74 & Male & Bed sores & 267 & Insulin \\
\hline 26 & 66 & Female & Diabetic foot & 360 & Insulin \\
\hline 27 & 48 & Male & Diabetic foot & 270 & Insulin \\
\hline 28 & 74 & Male & Diabetic foot & 215 & Insulin \\
\hline 29 & 62 & Male & Diabetic foot & 220 & Insulin \\
\hline 30 & 58 & Male & Diabetic foot & 580 & Insulin \\
\hline 31 & 58 & Female & Diabetic foot & 400 & Insulin \\
\hline 32 & 60 & Female & Bed sores & 300 & Insulin \\
\hline 33 & 73 & Male & Wound & 260 & Insulin \\
\hline 34 & 60 & Male & Diabetic foot & 350 & Insulin \\
\hline 35 & 56 & Male & Diabetic foot & 300 & Insulin \\
\hline 36 & 57 & Male & Wound & 120 & Tablet \\
\hline 37 & 72 & Male & Diabetic foot & 190 & Insulin \\
\hline 38 & 70 & Male & Diabetic foot & 180 & Tablet \\
\hline 39 & 60 & Female & Diabetic foot & 376 & Insulin \\
\hline 40 & 58 & Female & Bed sores & 280 & Insulin \\
\hline 41 & 75 & Female & Wound & 200 & Insulin \\
\hline 42 & 66 & Female & Diabetic foot & 250 & Insulin \\
\hline 43 & 60 & Female & Diabetic foot & 150 & Tablet \\
\hline 44 & 54 & Female & Diabetic foot & 270 & Insulin \\
\hline 45 & 58 & Female & Diabetic foot & 350 & Insulin \\
\hline 46 & 62 & Male & Diabetic foot & 200 & Insulin \\
\hline 47 & 50 & Male & Diabetic foot & 160 & Tablet \\
\hline 48 & 44 & Male & Wound & 140 & Tablet \\
\hline 49 & 62 & Male & Diabetic foot & 220 & Insulin \\
\hline 50 & 66 & Male & Diabetic foot & 200 & Insulin \\
\hline
\end{tabular}


Table (2): Antibiotics susceptibility test against bacterial isolates.

\begin{tabular}{|c|c|c|c|c|c|c|c|c|c|c|}
\hline \multirow{2}{*}{$\begin{array}{c}\text { No of } \\
\text { bacterial } \\
\text { isolates }\end{array}$} & \multirow[b]{2}{*}{$\begin{array}{c}\text { IPM } \\
10\end{array}$} & \multirow[b]{2}{*}{$\begin{array}{c}\text { AMC } \\
\mathbf{3 0}\end{array}$} & \multicolumn{5}{|c|}{ Antibiotics with IZDs mm } & \multirow{2}{*}{$\mathbf{R}$} & \multirow{2}{*}{$\mathbf{I}$} & \multirow{2}{*}{$\mathbf{S}$} \\
\hline & & & $\begin{array}{c}\text { NOR } \\
10\end{array}$ & $\begin{array}{c}\text { FOX } \\
\mathbf{3 0}\end{array}$ & N30 & F300 & $\begin{array}{c}\text { FEP } \\
\mathbf{3 0}\end{array}$ & & & \\
\hline 1 & $30 \mathrm{~S}$ & $28 \mathrm{~S}$ & $22 \mathrm{~S}$ & $17 \mathrm{R}$ & $18 \mathrm{~S}$ & $18 \mathrm{~S}$ & $8 \mathrm{R}$ & 2 & - & 5 \\
\hline 2 & $24 \mathrm{~S}$ & $35 \mathrm{~S}$ & $\mathrm{R}$ & $\mathrm{R}$ & $12 \mathrm{R}$ & $22 \mathrm{~S}$ & $13 \mathrm{R}$ & 3 & - & 4 \\
\hline 3 & $17 \mathrm{~S}$ & $\mathrm{R}$ & $17 \mathrm{~S}$ & $\mathrm{R}$ & $\mathrm{R}$ & $12 \mathrm{R}$ & $\mathrm{R}$ & 5 & - & 2 \\
\hline 4 & $15 \mathrm{I}$ & $\mathrm{R}$ & $17 \mathrm{~S}$ & $\mathrm{R}$ & $\mathrm{R}$ & $11 \mathrm{R}$ & $\mathrm{R}$ & 5 & 1 & 1 \\
\hline 5 & $24 \mathrm{~S}$ & $\mathrm{R}$ & $\mathrm{R}$ & $\mathrm{R}$ & $\mathrm{R}$ & $\mathrm{R}$ & $\mathrm{R}$ & 6 & - & 1 \\
\hline 6 & $25 \mathrm{~S}$ & $\mathrm{R}$ & $16 \mathrm{I}$ & $18 \mathrm{R}$ & $8 \mathrm{R}$ & $\mathrm{R}$ & $\mathrm{R}$ & 5 & 1 & 1 \\
\hline 7 & $18 \mathrm{~S}$ & $\mathrm{R}$ & $14 \mathrm{I}$ & $11 \mathrm{R}$ & $10 \mathrm{R}$ & $\mathrm{R}$ & $\mathrm{R}$ & 5 & 1 & 1 \\
\hline 8 & $23 \mathrm{~S}$ & $21 \mathrm{~S}$ & $\mathrm{R}$ & $\mathrm{R}$ & $\mathrm{R}$ & $21 \mathrm{~S}$ & $\mathrm{R}$ & 4 & - & 3 \\
\hline 9 & $25 \mathrm{~S}$ & $\mathrm{R}$ & $17 \mathrm{~S}$ & $14 \mathrm{R}$ & $18 \mathrm{~S}$ & $\mathrm{R}$ & $\mathrm{R}$ & 4 & - & 3 \\
\hline 10 & $27 \mathrm{~S}$ & $28 \mathrm{~S}$ & $18 \mathrm{~S}$ & $\mathrm{R}$ & $15 \mathrm{I}$ & $23 \mathrm{~S}$ & $\mathrm{R}$ & 2 & 1 & 4 \\
\hline 11 & $33 \mathrm{~S}$ & $15 \mathrm{R}$ & $21 \mathrm{~S}$ & $12 \mathrm{R}$ & $9 \mathrm{R}$ & $17 \mathrm{~S}$ & $\mathrm{R}$ & 4 & - & 3 \\
\hline 12 & $33 \mathrm{~S}$ & $26 \mathrm{~S}$ & $19 \mathrm{~S}$ & $26 \mathrm{~S}$ & $20 \mathrm{~S}$ & $12 \mathrm{R}$ & $14 \mathrm{R}$ & 2 & - & 5 \\
\hline 13 & $21 \mathrm{~S}$ & $15 \mathrm{R}$ & $\mathrm{R}$ & $\mathrm{R}$ & $\mathrm{R}$ & $17 \mathrm{~S}$ & $\mathrm{R}$ & 5 & -- & 2 \\
\hline 14 & $28 \mathrm{~S}$ & $10 \mathrm{R}$ & $\mathrm{R}$ & $16 \mathrm{R}$ & $15 \mathrm{I}$ & $18 \mathrm{~S}$ & $\mathrm{R}$ & 4 & 1 & 2 \\
\hline 15 & $25 \mathrm{~S}$ & $10 \mathrm{R}$ & $\mathrm{R}$ & $14 \mathrm{R}$ & $16 \mathrm{I}$ & $17 \mathrm{~S}$ & $\mathrm{R}$ & 4 & 1 & 2 \\
\hline 16 & $35 \mathrm{~S}$ & $20 \mathrm{~S}$ & $15 \mathrm{I}$ & $\mathrm{R}$ & $\mathrm{R}$ & $\mathrm{R}$ & $\mathrm{R}$ & 4 & 1 & 2 \\
\hline 17 & $\mathrm{R}$ & $14 \mathrm{R}$ & $23 \mathrm{~S}$ & $\mathrm{R}$ & $\mathrm{R}$ & $22 \mathrm{~S}$ & $\mathrm{R}$ & 5 & - & 2 \\
\hline 18 & $27 \mathrm{~S}$ & $25 \mathrm{~S}$ & $\mathrm{R}$ & $\mathrm{R}$ & $\mathrm{R}$ & $17 \mathrm{~S}$ & $\mathrm{R}$ & 4 & - & 3 \\
\hline 19 & $\mathrm{R}$ & $\mathrm{R}$ & $18 \mathrm{~S}$ & $\mathrm{R}$ & $\mathrm{R}$ & $\mathrm{R}$ & $\mathrm{R}$ & 6 & - & 1 \\
\hline 20 & $24 \mathrm{~S}$ & $\mathrm{R}$ & $\mathrm{R}$ & $\mathrm{R}$ & $\mathrm{R}$ & $\mathrm{R}$ & $\mathrm{R}$ & 6 & - & 1 \\
\hline 21 & $30 \mathrm{~S}$ & $15 \mathrm{R}$ & $\mathrm{R}$ & $\mathrm{R}$ & $13 \mathrm{R}$ & $\mathrm{R}$ & $\mathrm{R}$ & 6 & - & 1 \\
\hline 22 & $27 \mathrm{~S}$ & $\mathrm{R}$ & $\mathrm{R}$ & $\mathrm{R}$ & $\mathrm{R}$ & $\mathrm{R}$ & $\mathrm{R}$ & 6 & - & 1 \\
\hline 23 & $35 \mathrm{~S}$ & $32 \mathrm{~S}$ & $26 \mathrm{~S}$ & $24 \mathrm{~S}$ & $\mathrm{R}$ & $20 \mathrm{~S}$ & $18 \mathrm{R}$ & 2 & - & 5 \\
\hline 24 & $17 \mathrm{~S}$ & $\mathrm{R}$ & $\mathrm{R}$ & $\mathrm{R}$ & $\mathrm{R}$ & $\mathrm{R}$ & $\mathrm{R}$ & 6 & - & 1 \\
\hline 25 & $34 \mathrm{~S}$ & $\mathrm{R}$ & $31 \mathrm{~S}$ & $\mathrm{R}$ & $18 \mathrm{I}$ & $\mathrm{R}$ & $\mathrm{R}$ & 4 & 1 & 2 \\
\hline 26 & $30 \mathrm{~S}$ & $27 \mathrm{~S}$ & $24 \mathrm{~S}$ & $22 \mathrm{~S}$ & $16 \mathrm{I}$ & $24 \mathrm{~S}$ & $18 \mathrm{R}$ & 1 & 1 & 5 \\
\hline 27 & $18 \mathrm{~S}$ & $\mathrm{R}$ & $\mathrm{R}$ & $\mathrm{R}$ & $18 \mathrm{~S}$ & $14 \mathrm{R}$ & $\mathrm{R}$ & 5 & - & 2 \\
\hline 28 & $35 \mathrm{~S}$ & $24 \mathrm{~S}$ & $\mathrm{R}$ & $\mathrm{R}$ & $\mathrm{R}$ & $20 \mathrm{~S}$ & $\mathrm{R}$ & 4 & - & 3 \\
\hline 29 & $30 \mathrm{~S}$ & $13 \mathrm{R}$ & $13 \mathrm{I}$ & $\mathrm{R}$ & $\mathrm{R}$ & $\mathrm{R}$ & $\mathrm{R}$ & 5 & 1 & 1 \\
\hline 30 & $\mathrm{R}$ & $\mathrm{R}$ & $\mathrm{R}$ & $\mathrm{R}$ & $\mathrm{R}$ & $\mathrm{R}$ & $\mathrm{R}$ & 7 & - & - \\
\hline 31 & $\mathrm{R}$ & $\mathrm{R}$ & $\mathrm{R}$ & $\mathrm{R}$ & $\mathrm{R}$ & $\mathrm{R}$ & $\mathrm{R}$ & 7 & - & - \\
\hline 32 & $28 \mathrm{~S}$ & $\mathrm{R}$ & $\mathrm{R}$ & $\mathrm{R}$ & $\mathrm{R}$ & $\mathrm{R}$ & $\mathrm{R}$ & 6 & - & 1 \\
\hline 33 & $20 \mathrm{~S}$ & $\mathrm{R}$ & $18 \mathrm{~S}$ & $\mathrm{R}$ & $16 \mathrm{I}$ & $\mathrm{R}$ & $16 \mathrm{R}$ & 5 & 1 & 1 \\
\hline 34 & $12 \mathrm{R}$ & $\mathrm{R}$ & $17 \mathrm{~S}$ & $\mathrm{R}$ & $\mathrm{R}$ & $\mathrm{R}$ & $\mathrm{R}$ & 6 & - & 1 \\
\hline 35 & $\mathrm{R}$ & $\mathrm{R}$ & $\mathrm{R}$ & $\mathrm{R}$ & $\mathrm{R}$ & $\mathrm{R}$ & $\mathrm{R}$ & 7 & - & - \\
\hline 36 & $16 \mathrm{~S}$ & $\mathrm{R}$ & $18 \mathrm{~S}$ & $\mathrm{R}$ & $18 \mathrm{~S}$ & $15 \mathrm{R}$ & $\mathrm{R}$ & 4 & - & 3 \\
\hline 37 & $26 \mathrm{~S}$ & $\mathrm{R}$ & $\mathrm{R}$ & $24 \mathrm{~S}$ & $\mathrm{R}$ & $\mathrm{R}$ & $\mathrm{R}$ & 5 & - & 2 \\
\hline 38 & $18 \mathrm{~S}$ & $\mathrm{R}$ & $16(\mathrm{I})$ & $\mathrm{R}$ & $\mathrm{R}$ & $\mathrm{R}$ & $\mathrm{R}$ & 5 & 1 & 1 \\
\hline 39 & $30 \mathrm{~S}$ & $22 \mathrm{~S}$ & $22 \mathrm{~S}$ & $20 \mathrm{~S}$ & $11 \mathrm{R}$ & $16 \mathrm{I}$ & $14 \mathrm{R}$ & 2 & 1 & 4 \\
\hline 40 & $12 \mathrm{R}$ & $\mathrm{R}$ & $\mathrm{R}$ & $\mathrm{R}$ & $\mathrm{R}$ & $\mathrm{R}$ & $\mathrm{R}$ & 7 & - & 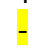 \\
\hline $\mathbf{S}$ & 32 & 11 & 16 & 5 & 5 & 12 & - & & & \\
\hline I & 1 & - & 5 & - & 6 & 1 & - & & & \\
\hline $\mathbf{R}$ & 7 & 29 & 19 & 35 & 29 & 26 & 40 & & & \\
\hline
\end{tabular}


Table (3): Morphological characteristics and biochemical identification of bacterial isolates.

\begin{tabular}{lllll}
\hline Test & P. aeruginosa $\boldsymbol{D F}$ & K. pneumonia $\boldsymbol{D}$ & K. pneumonia $\boldsymbol{B S}$ & S. aureus $\boldsymbol{W}$ \\
\hline Gram stain & $-\mathrm{ve}$ & $-\mathrm{ve}$ & $-\mathrm{ve}$ & $+\mathrm{ve}$ \\
Motility & $+\mathrm{ve}$ & $-\mathrm{ve}$ & $-\mathrm{ve}$ & $-\mathrm{ve}$ \\
Growth on MacConky & $+\mathrm{ve}$ & $+\mathrm{ve}$ & $+\mathrm{ve}$ & $\mathrm{ND}$ \\
Shape of cell & Rod shape & Rod shape & Rod shape & Cocci shape \\
Sugar fermentation reactions & $+\mathrm{ve}$ & & & \\
D-Glucose & $-\mathrm{ve}$ & $+\mathrm{AG}$ & $+\mathrm{AG}$ & $\mathrm{ND}$ \\
Lactose & $-\mathrm{ve}$ & $+\mathrm{ve}$ & $+\mathrm{ve}$ \\
Sucrose & $+\mathrm{ve}$ & $+\mathrm{ve}$ & $\mathrm{ND}$ \\
Maltose & $-\mathrm{ve}$ & $+\mathrm{ve}$ & $+\mathrm{ve}$ & $\mathrm{ND}$ \\
D-sorbitol & $-\mathrm{ve}$ & $+\mathrm{ve}$ & $+\mathrm{ve}$ & $\mathrm{ND}$ \\
D-Mannitol & $+\mathrm{ve}$ & $+\mathrm{ve}$ & $+\mathrm{ve}$ & $+\mathrm{ve}$ \\
D-Mannose & $-\mathrm{ve}$ & $+\mathrm{ve}$ & $+\mathrm{ve}$ & $\mathrm{ND}$ \\
Specific biochemical reactions & $\mathrm{ND}$ & & \\
B-hemolysis & $\mathrm{ND}$ & $\mathrm{ND}$ & $\mathrm{ND}$ & $+\mathrm{ve}$ \\
Catalase & $+\mathrm{ve}$ & $+\mathrm{ve}$ & $+\mathrm{ve}$ & $+\mathrm{ve}$ \\
Coagulase & $\mathrm{ND}$ & $\mathrm{ND}$ & $\mathrm{ND}$ & $+\mathrm{ve}$ \\
Oxidase & $+\mathrm{ve}$ & $-\mathrm{ve}$ & $-\mathrm{ve}$ & $-\mathrm{ve}$ \\
Indole & $-\mathrm{ve}$ & $-\mathrm{ve}$ & $-\mathrm{ve}$ & $-\mathrm{ve}$ \\
Citrate & $-\mathrm{ve}$ & $+\mathrm{ve}$ & $-\mathrm{ve}$ & $-\mathrm{ve}$ \\
H2S & $-\mathrm{ve}$ & $-\mathrm{ve}$ & $-\mathrm{ve}$ & $+\mathrm{ve}$ \\
Urease & $+\mathrm{ve}$ & $+\mathrm{ve}$ & $+\mathrm{ve}$ & \\
\hline
\end{tabular}

Table (4): Determination MIC to three antibiotics for selected bacterial isolates.

\begin{tabular}{|c|c|c|c|c|c|c|c|c|c|c|}
\hline \multirow{2}{*}{ Bacterial isolates } & \multirow{2}{*}{$\begin{array}{l}\text { Antibioti } \\
\text { c }\end{array}$} & \multicolumn{9}{|c|}{$\begin{array}{c}\text { Different concentrations of antibiotic } \\
\text { and IZD }(\mathrm{mm})\end{array}$} \\
\hline & & $100 \mathrm{mgl}$ & $50 \mathrm{mgl}$ & $5 \mathrm{mgl}$ & $\begin{array}{l}\mathbf{5 0 0} \\
\mu g 】\end{array}$ & $250 \mu \mathrm{gl}$ & $125 \mu \mathrm{gl}$ & $12.5 \mu \mathrm{gl}$ & $\begin{array}{l}1.25 \\
\mu g \backslash l\end{array}$ & $\begin{array}{c}0.125 \\
\mu g l\end{array}$ \\
\hline P. aeruginosa $D F$ & & $16 \pm 4.54$ & $13 \pm 3.67$ & $7 \pm 2.64$ & 0 & 0 & 0 & 0 & 0 & 0 \\
\hline K. pneumonia $D$ & IPM & $23 \pm 2.54$ & $21 \pm 1.00$ & $16 \pm 2.00$ & $9 \pm 2.64$ & $7 \pm 0.64$ & 0 & 0 & 0 & 0 \\
\hline K. pneumonia BS & IPM & $26 \pm 2.64$ & $25 \pm 0.74$ & $20 \pm 2.43$ & $15 \pm 1.00$ & $13 \pm 2.64$ & $11 \pm 3.54$ & $9 \pm 2.00$ & 0 & 0 \\
\hline S. aureus W & & $18 \pm 1.34$ & $16 \pm 2.64$ & $15 \pm 3.54$ & $14 \pm 1.23$ & $14 \pm 2.64$ & $13 \pm 1.00$ & $13 \pm 1.45$ & $11 \pm 2.64$ & 0 \\
\hline P. aeruginosa $D F$ & & $25 \pm 2.00$ & $20 \pm 3.76$ & $13 \pm 1.67$ & 0 & 0 & 0 & 0 & 0 & 0 \\
\hline K. pneumonia $D$ & NOR & $18 \pm 1.54$ & $16 \pm 1.64$ & $11 \pm 2.64$ & 0 & 0 & 0 & 0 & 0 & 0 \\
\hline K. pneumonia BS & NOR & $20 \pm 4.73$ & $16 \pm 0.64$ & $11 \pm 4.64$ & 0 & 0 & 0 & 0 & 0 & 0 \\
\hline S. aureus $W$ & & $16 \pm 2.64$ & $15 \pm 1.66$ & $13 \pm 0.84$ & 0 & 0 & 0 & 0 & 0 & 0 \\
\hline P. aeruginosa $D F$ & & $11 \pm 3.73$ & 0 & 0 & 0 & 0 & 0 & 0 & 0 & 0 \\
\hline K. pneumonia $D$ & & $17 \pm 1.64$ & 0 & 0 & 0 & 0 & 0 & 0 & 0 & 0 \\
\hline K. pneumonia $B S$ & $\mathbf{F}$ & $19 \pm 4.32$ & $18 \pm 1.64$ & $16 \pm 1.00$ & $18 \pm 2.64$ & 0 & 0 & 0 & 0 & 0 \\
\hline S. aureus $W$ & & $16 \pm 2.64$ & 0 & 0 & 0 & 0 & 0 & 0 & 0 & 0 \\
\hline
\end{tabular}

Table (5): MBC to three antibiotics for selected bacterial isolates.

\begin{tabular}{lll}
\hline \multicolumn{1}{c}{ Bacterial isolates } & Antibiotic & MBC \\
\multirow{3}{*}{ K. pneumonia D } & IPM & $5 \mathrm{mg} \backslash \mathrm{ml}$ \\
& NOR & ND \\
& F & ND \\
\hline \multirow{2}{*}{. aeruginosa DF } & IPM & $100 \mathrm{mg} \backslash \mathrm{ml}$ \\
& NOR & ND \\
& F & ND \\
S. aureus W & IPM & $100 \mathrm{mg} \backslash \mathrm{ml}$ \\
& NOR & $200 \mathrm{mg} \backslash \mathrm{ml}$ \\
& F & $100 \mathrm{mg} \backslash \mathrm{ml}$ \\
\hline \multirow{2}{*}{ pneumonia BS } & IPM & $100 \mathrm{mg} \backslash \mathrm{l}$ \\
& NOR & $5 \mathrm{mg} \backslash \mathrm{l}$ \\
& F & $5 \mathrm{mg} \backslash \mathrm{ml}$ \\
\hline
\end{tabular}


Table (6): Antibacterial activities of boiling water plant extracts on bacterial isolates.

\begin{tabular}{lllll}
\hline \multirow{2}{*}{ Plant extracts } & \multicolumn{4}{c}{ IZD $(\mathbf{m m})$} \\
\cline { 2 - 5 } & P. aeruginosa & K. pneumonia 1 & K. pneumonia 2 & S. aureus \\
\hline Clove & $8 \pm 2.51$ & 0 & 0 & $10 \pm 2.64$ \\
Garlic & 0 & $10 \pm 0.47$ & 0 & $7 \pm 0.64$ \\
Cinnamon & $8 \pm 1.00$ & $10 \pm 1.00$ & $13 \pm 1.00$ & $12 \pm 1.00$ \\
Moringa green leaves & 0 & 0 & 0 & 0 \\
Moringa dry leaves & 0 & 0 & 0 & 0 \\
Moringa seeds & 0 & 0 & 0 & 0 \\
Ginger & 0 & 0 & 0 & 0 \\
Aloe vera & 0 & 0 & 0 & 0 \\
\hline
\end{tabular}

\section{DISCUSSION}

In clinical practice worldwide, infectious diseases related to antibiotic resistance is a major threat to human health. Antibiotic resistance can affect anyone, of any age (Khalifa et al., 2019). So, there is a need for the discover of safe and nontoxic natural products with the antimicrobial capacity to be utilized as alternative medication in general health. Out of 50 bacterial isolates, 4 only were resistant to all antibiotic groups this supported later work in this respect (Abdel-Shafi, 2013). Selected multidrug resistant bacteria of this study were identified herein as belonging to $S$. aureus, $P$. aeruginosa, K. pneumonia, and later published work showed the prevalence of similar drug resistant bacteria (Abeer et al., 2020).

Antibiotic susceptibility testing is the measurement of the susceptibility of bacteria to the antibiotic. It is used due to the resistance of bacteria to some antibiotics. (Leekha et al., 2011).

From our results, the most effective antibiotic against the isolated clinical bacteria was imipenem, this agreement with the study of Helmut et al., (1985) they showed that the activity of imipenem is high against $S$. aureus, streptococci, Bacteroides, $P$. aeruginosa and $S$. epidermidis. Imipenem was previously reported to be the most effective and similar effectiveness of these antibiotic were also reported (Abeer $\boldsymbol{e t}$ al., 2020).
Many natural plant extracts used as flavoring and seasoning agents in food have been used therapeutically for centuries (Vinoth et al., 2011). Antibacterial activities of cinnamon, onion garlic, and cloves and their active compounds have been studied since the end of the last century (Jagadeesh et al., 2011). Cinnamon contains mainly cinnamaldehyde $(50.5 \%)$, cinnamyl acetate $(8.7 \%)$, eugenol (4.7\%), methoxy cinnamaldehyde (MOCA) and cinnamic acid (Charu et al., 2008). Allicin is the active compound present in garlic, which is a thiosuffinate compound reported for its highest antimicrobial activity (Chung et al., 2007). Clove is characterized by the presence of eugenol and other phenolic compounds that Characterized by their antimicrobial activity (Mittal et al., 2014).

In our study, six plant extracts were tested for their inhibitory activities against MDR pathogenic bacteria. Our results showed that the boiling water plant extracts of cinnamon, clove and garlic, had antibacterial activity against MDR bacteria, while, moringa green leaves, moringa dry leaves and moringa seeds had no antibacterial activity. Our results agree with Avijit $\boldsymbol{e t}$ al., (2018), they showed that the ethanol extract of garlic, clove and cinnamon was highly active against $S$. aureus, Shigella spp., B. cereus Serratia spp., E. coli, S. typhi and Acinetobacter spp. and Klebsiella spp. Furthermore, Maha et al., (2012), demonstrated that alcoholic extract of clove, cinnamon and thyme showed antibacterial activity against 
S. aureus, B. subtilis, E. coli, $P$. aeruginosa, $S$. typhi, C. albicans and A. niger.

\section{CONCLUSIONS}

According to the obtained results, it can be concluded that cinnamon could be used as an antibacterial agent. It can be efficiently and successfully used as a safe, natural product. It can be prepared with a low cost.

\section{REFERENCES}

Abdel-Azim, N.S.; Shams, K.A.; Shahat, A.A.; El Missiry, M.M.; Ismail, S.I.; Hammouda, F.M. (2011): Egyptian herbal drug industry: challenges and future prospects. Res J Med Plant, 5: 136-144.

Abdel-Shafi, S.; Osman, A.; Enan, G.; ElNemer, M.; Sitohy, M. (2016): Antibacterial activity of methylated egg white proteins against pathogenic $\mathrm{G}^{+}$and $\mathrm{G}^{-}$bacteria matching antibiotics. Springer Plus, 5 (1) 983.

Abdel-Shafi, S.; Ouda, S.M.; Elbalat, I.; Enan, G. (2013): Characterization and Identification of Multidrug Resistant Bacteria from Some Egyptian Patients. Biotechnology; 12:65-73.

Abeer, F.; Mohamed, F.; and Ahmed, S. (2020): Identification of multidrug resistant bacterial isolates from Egyptian hospitals environments and molecular detection of their resistance genes. Bioscience research.17 (3): 2015-2024.

Andrews, J.M. (2001): Determination of minimum inhibitory concentrations. Antimicrob. Chemother. 48(6):5-16.

Avijit, B.; Maruf, A.; Tamanna, Z.; Suvamoy, D. (2018): Antibacterial Activity of Allium Sativum, Syzygium Aromaticum, and Cinnamomum Zeylanicum against Food Borne Pathogens in Vitro, Journal of Pharmacy and Biological Sciences, 13: 68-73.
Bauer, A.W.; Kirby, W.M.M.; Sherris, J.C.; and Turk, M. (1966): Antibiotic susceptibility testing by a standerized single disk method. Am. J. of clin. Pathol, (45): 493-496.

Benson, H.J. (1998): Microscope Slide Techniques (Bacterial Morphology). In: Microbiological Applications Laboratory Manual in Ge010neral Microbiology. $7^{\text {th }}$ ed. Boston, California St. Louis Missouri, 49-70.

Bogers, R.J.; Craker, L.E.; Lange, D. (2006): Medicinal and aromatic plants: agricultural, commercial, ecological, legal, pharmacological and social aspects. Berlin, Heidelberg: Springer, 17: 1573-4544.

Charu, G.; Amar, P.; Ramesh, C.; and Archana, K. (2008): Comparative analysis of the antimicrobial activity of cinnamon oil and cinnamon extract on some food-borne microbes. African Journal of Microbiology Research, 2(9), 247-251.

Chung, I.; Kwon, S.H.; Shim, S.-T.; And Kyung, K.H. (2007): Journal of food science, 72, M434-M440.

Cloete, T.E.; (2003): Resistance mechanisms of bacteria to antimicrobial compounds. Int. Biodeterant. Biodegradat, 51: 277-282.

CLSI, Clinical and Laboratory Standards Institute (2012): Methods for Dilution Antimicrobial Susceptibility Tests for Bacteria that Grow Aerobically, Approved Standard, 9th ed., CLSI document M07-A9. Clinical and Laboratory Standards Institute, 950 West Valley Road, Suite 2500, Wayne, Pennsylvania 19087, USA.

Collee, J.G.; and Marr, W. (1989): Culture containers and culture media. In "Practical Medical Microbiology", $13^{\text {th }}$ ed. Vol. (2). Collee, J. G; Duguid, J. P; Fraser, A. G; and Marmion, B. P. (eds), Churchill Livingstone. New York. 100-120. 
Collee, J.G.; Marr, W.; Fraser, A.G.; Marmion, B.P.; and Simmons, A. (1996): Specimen collection, culture containers and media.: Mackie and Mac Carthey Practical medical microbiology. Churchill Livingstone, New York, London, Tokyo. 95-111.

Egyptain Pharma Copoeia (1984): The Pharmaceutical Press, Vol. I. El Atki, Y.; Aouam, I.; El Kamari, F.; Taroq, A.; Nayme, K.; Timinouni, M.; Lyoussi, B.; and Abdellaoui, A. (2019): Antibacterial activity of cinnamon essential oils and their synergistic potential with antibiotics. J Adv Pharm Technol Res. 10(2):63-67.

El Atki, Y.; Aouam, I.; El Kamari, F.; Taroq, A.; Nayme, K.; Timinouni, M.; Lyoussi, B.; and Abdellaoui, A. (2019): Antibacterial activity of cinnamon essential oils and their synergistic potential with antibiotics. J Adv Pharm Technol Res, 10(2):63-67.

Eucast (2003): Determination of minimum inhibitory concentrations (MICs) of antibacterial agents by broth dilution. Clin. Microbiol. Infect. 6(9):509-515.

Farland, M.C. (1987): Standardization of bacterial culture for disc diffusion assay. Journal of American Medical Association, 49: 1176-1178.

Harvey, R.A.; and Champe, D.C. (2012): Lippincott's Illustrated Reviews in Microbiology. Lippincott Williams and Wilkins, USA, 19-22.

Helmut, K.; Lynn, G.; Jon, G.; Frederick, M. (1985): Antibacterial Activity of Imipenem: The First Thienamycin Antibiotic. Reviews of Infectious Diseases, 7(3): 389-410.

Izzo, A. A.; Di Garlo, G. Biscardi, D. De Fusco, R. Mascolo, N. Borrell, F.; Capasso, F.; Fasulo, M. P. and Autore, G. (1995): "Biological screening of Italian medicinal plants for antibacterial activity". Phytotherapy. Research. 9, 281-286.
Jagadeesh, A.; Rupa, A.; Indumathi, J.; Srujan, R.; and Sravanthi, M. (2011): Research. 5. Study on the antimicrobial activity and minimum inhibitory concentration of essential oils of spices. Veterinary World, 4(7), 311-316.

Khalifa, H.O.; Soliman, A.M.; Ahmed, A.M.; et al. (2019): High Prevalence of Antimicrobial Resistance in GramNegative Bacteria Isolated from Clinical Settings in Egypt: Recalling for Judicious Use of Conventional Antimicrobials in Developing Nations. Microb Drug Resist, 25:371-385.

Koneman, E.W.; Allen, S.D.; Janda, W.M.; Schreckenberger, P.C.; and Winn, W.C. (1997): Introduction to microbiology part I: The role of the microbiology laboratory in the diagnosis of infection diseases. Guidelines to practice and management in "Color atlas and text book of diagnosis microbiology". Fifth edition. Philadelphia. 69-120.

Lambert, R.J.; Pearson, J. (2000): Susceptibility testing: Accurate and reproducible minimum inhibitory concentration (MIC) and noninhibitory concentration (NIC) values. J. Appl. Microbiol, 88(5):784-790.

Leekha, S.; Terrell, C.L.; Edson, R.S. (2011): General principles of antimicrobial therapy". Mayo Clinic Proceedings, 86 (2): 156-67.

Leon, J.; Rojo, E.; and Sanchezerrano, J. (2001): Wound signaling in plants. Journal of Experimental Botany, 52: 19.

Lorian, V. (2005): Antibiotics in laboratory medicine. Books in laboratory medicine. In: Lorian, V. editions (5th ed.), Published by Lippincott Williams \& Wilkins; P: 1-6. 
Maha, M.; Ismail, T.M.; Essam, A.F.M; and Fathia, E.M. (2012): Screening for the Antimicrobial Activities of Alcoholic and Aqueous Extracts of Some Common Spices in Egypt. International Journal of Microbiological Research 3 (3): 200207.

Mittal, M.; Gupta, N.; Parashar, P.; Mehra, P.; Khatri, M (2014): Phytochemical evaluation and pharmacological activity of syzygium aromaticum. Int J Pharm Sci, (6): 6772.

Owuama, C.I. (2015): Microbiology Laboratory manual. Microtrend Digital Press, Yola. ISBN 978-978-943-328-2.

Rita, B.; Jackie, R.; Donald, P. (2009): Differential Staining of Bacteria: Gram Stain. Current protocols in microbiology, 15: 1-8.
Robert, C. M. (2011): Discovering new antimicrobial agents. International Journal of Antimicrobial Agents, 37: 2-9.

Trujillo, M. E.; Goodfellow, M. (2012): Genus I. Brevibacterium Breed 1953a. 13AL emend. Colins, Jones, Keddie and Sneath 1980. In Bergeys Manual of Systematic Bacteriology, 2nd edn.vol. 5: The Actinobacteria, 685700 .

Vinoth, K.; Amala, B.; Veera, V.; Sudheer, C.; and Ramesh, N. (2010): Biopotency of Allium sativum. Linn (Garlic) in Lead Acetate Intoxicated Male Albino Rats. Der Pharmacia Sinica, 1 (2): 1-4.

Yamamoto, L.G. (2003): Inhibitory and bactericidal principles MIC\&MB. Journal of biological sciences. (4): 1-8. 